\title{
Hacia una unión económica más fuerte de la Unión Europea a través de la celebración de un cuestionado tratado internacional $^{*}$
}

\author{
María Isabel Martín de Llano \\ Profesora Contratada Doctora \\ Departamento de Derecho Político. Universidad Nacional de Educación a Distancia
}

Recibido: 01.10 .2012

Aceptado: 15.10 .2012

Resumen: Con el objetivo de conseguir una Unión Económica más fuerte, en marzo de 2012, veinticinco Estados miembros de la Unión Europea firmaron un Tratado internacional incluye un nuevo pacto presupuestario, una mayor coordinación de las políticas económicas y un refuerzo de los instrumentos de estabilización. La entrada en vigor de este Tratado genera grandes incertidumbres al haberse firmado al margen de los Tratados constitutivos de la Unión Europea.

Palabras Clave: Tratado Internacional, Pacto presupuestario, Gobernanza de la euro zona.

Abstract: To get a stronger economic union, twenty-five member States of the European Union signed in March 2012 an international Treaty that includes a new budget agreement, a greater coordination of economic policies and improved tools for the stabilization. The entry into force of this Treaty creates great uncertainty, as it has been signed without considering the founding treaties of the European Union.

Keywords: International Treaty, Budget agreement, Eurozone governance.

Sumario: 1. Introducción.-2. El Tratado de Estabilidad, Coordinación y Gobernanza en la Unión Económica y Monetaria.-3. Un Tratado intergubernamental en el seno de la Unión Europea.- - 4. El TECG en relación al Derecho e Instituciones de la Unión Europea.- 5. La Gobernanza de la zona del euro.-6. Conclusiones.

\section{INTRODUCCIÓN}

Uno de los objetivos de la Unión Europea era contar con una moneda única, algo que se consigue en 1999 con el euro. De conformidad con lo establecido en el Tratado de Funcionamiento de la Unión Europea (TFUE) la política monetaria se instrumenta a escala supranacional mientras que las

* Este trabajo se realiza en el marco del Proyecto de Investigación «Constitución y globalización: transfomaciones del Estado constitucional y constitucionalización de espacios supranacionales» DER 2009-10375 (Subprograma JURI), Ministerio de Ciencia e Innovación. 
políticas presupuestarias, financieras y estructurales son competencia, en gran medida, de los Gobiernos nacionales. Esto ha conllevado que aunque los pilares monetarios de la Unión eran fuertes no ha existido una regulación fiscal y financiera definida, ni un mecanismo de resolución de posibles crisis, ni un fondo de rescate para Estados miembros o instituciones financieras con problemas de liquidez.

La crisis económica mundial que estalló en 2008 y que continúa agravándose cada vez más en determinados países de la Unión ha obligado a que los Estados miembros se planteen la necesidad de adoptar una serie de medidas normativas destinadas a garantizar la estabilidad y la permanencia del euro como moneda única.

Tras una serie de actuaciones en este sentido (la creación del Fondo de rescate temporal europeo-vigente hasta 2013-, el Mecanismo Europeo de Estabilidad -de carácter permanente- y el Pacto por el Euro Plus -acordado con el objetivo de potenciar la productividad y competitividad de las economías de los países miembros y con ello el crecimiento-), el Consejo Europeo celebrado el 9 diciembre 2011 con el fin de consolidar la confianza en la fortaleza de la economía europea consideró necesario aplicar plenamente la nueva gobernanza económica de la Unión; en este sentido, ese mismo día los Jefes de Estado y de Gobierno de la zona del euro aprobaron una Declaración en la que se comprometieron a celebrar un acuerdo internacional al margen de los Tratados constitutivos de la Unión Europea con el objetivo de avanzar hacia una unión económica más fuerte. Para ello se pretende actuar en dos direcciones: un nuevo pacto presupuestario y una coordinación reforzada de las políticas económicas; y el desarrollo de las herramientas de estabilización para hacer frente a los desafíos a corto plazo.

Los Estados firmantes de esta Declaración consideraron que la estabilidad e integridad de la Unión Económica y Monetaria y de la Unión Europea en su conjunto requieren nuevos avances cualitativos hacia una auténtica «unión de estabilidad presupuestaria» en la zona del euro, siendo necesario la existencia de un pilar económico fuerte cuya base será una gobernanza reforzada para promover la disciplina presupuestaria y una integración más profunda del mercado interior, así como un crecimiento más fuerte, una mayor competitividad y la cohesión social. Para ello se comprometieron a establecer una nueva norma presupuestaria que se introducirá en los ordenamientos jurídicos de los Estados miembros integrándola en la Constitución o en una norma de nivel equivalente y que contendrá un mecanismo de corrección automático que se activará en caso de desviación.

Ante la falta de unanimidad entre los Estados miembros, los Jefes de Estado y de Gobierno de la zona del euro acordaron adoptar las medidas previstas en la mencionada Declaración mediante un acuerdo internacional cuyo contenido, en un futuro, debería incorporarse a los Tratados de la Unión. 


\section{EL TRATADO DE ESTABILIDAD, COORDINACIÓN Y GOBER- NANZA EN LA UNIÓN ECONÓMICA Y MONETARIA}

El 2 de marzo de 2012, veinticinco Estados miembros de la Unión Europea ${ }^{1}$ firmaron el "Tratado de Estabilidad, Coordinación y Gobernanza en la Unión Económica y Monetaria» cuyo objetivo principal es promover la disciplina presupuestaria con el fin de conseguir la sostenibilidad financiera de los Estados de la zona euro. En este Tratado las Partes Contratantes se comprometen a aplicar, entre otras, las siguientes normas:

a) la situación presupuestaria de las administraciones públicas de cada Parte Contratante será de equilibrio o de superávit;

b) la norma anterior se considerará respetada si el saldo estructural anual de las administraciones públicas ${ }^{2}$ alcanza el objetivo nacional específico a medio plazo, definido en el Pacto de Estabilidad y Crecimiento revisado, con un límite inferior de déficit estructural del $0,5 \%$ del producto interior bruto a precios de mercado. Las Partes Contratantes garantizarán la rápida convergencia hacia su respectivo objetivo a medio plazo. El calendario para lograr dicha convergencia lo propondrá la Comisión Europea tomando en consideración los riesgos específicos para la sostenibilidad de cada país. Los avances hacia el objetivo a medio plazo y su observancia serán objeto de una evaluación global que tome como referencia el saldo estructural e incluya un análisis del gasto excluidas las medidas discrecionales relativas a los ingresos, en consonancia con el Pacto de Estabilidad y Crecimiento revisado;

c) las Partes Contratantes podrán desviarse temporalmente de su objetivo a medio plazo respectivo o de la senda de ajuste hacia dicho objetivo únicamente en las circunstancias excepcionales ${ }^{3}$;

d) cuando la proporción entre la deuda pública y el producto interior bruto a precios de mercado esté muy por debajo del $60 \%$ y los riesgos para la sostenibilidad a largo plazo de las finanzas públicas sean bajos, el límite inferior del objetivo a medio podrá alcanzar un

${ }^{1}$ Todos menos la República Checa y el Reino Unido.

${ }^{2}$ El mismo Tratado define el «saldo estructural anual de las administraciones públicas» como el saldo anual ajustado en función del ciclo, una vez excluidas las medidas puntuales y las de carácter temporal.

${ }^{3}$ El mismo Tratado entiende por «circunstancias excepcionales» aquel acontecimiento inusual que esté fuera del control de la Parte Contratante afectada y tenga una gran incidencia en la situación financiera de las administraciones públicas o aquellos períodos de grave recesión económica a tenor del Pacto de Estabilidad y Crecimiento revisado, siempre que la desviación temporal de la Parte Contratante afectada no ponga en peligro la sostenibilidad presupuestaria a medio plazo. 
déficit estructural máximo del $1 \%$ del producto interior bruto a precios de mercado;

e) en el caso de que se observen desviaciones significativas se activará automáticamente un mecanismo corrector con el fin de corregir las desviaciones en un plazo de tiempo determinado;

f) la Parte Contratante que sea objeto de un procedimiento de déficit excesivo establecerá un programa de colaboración presupuestaria y económica en el que incluya una descripción pormenorizada de las reformas estructurales que se deberán adoptar y aplicar a fin de garantizar una corrección efectiva y duradera de su déficit;

g) sobre la base de la coordinación de las políticas económicas definida en el Tratado de Funcionamiento de la Unión Europea, las Partes Contratantes se comprometen a cooperar en aras de una política económica que fomente el correcto funcionamiento de la unión económica y monetaria y el crecimiento económico mediante una convergencia y competitividad reforzadas.

De esta forma se considera que el Tratado facilitará una convergencia más rápida hacia la consecución de los objetivos presupuestarios a medio plazo de los distintos países de la zona euro. Para ello se requiere que la Comisión Europea proponga calendarios de convergencia ambiciosos y vinculantes cuyo cumplimiento permita recuperar la confianza en la sostenibilidad fiscal de los Estados respectivos y restablecer la credibilidad de sus políticas presupuestarias.

Por otra parte, al establecerse un mecanismo corrector automático que se basará en principios comunes propuestos por la Comisión Europea, es esencial que ésta establezca unos principios específicos, estrictos y vinculantes de manera que dicho mecanismo corrector equivalga a un «freno a la deuda» y contribuya a corregir y evitar unas finanzas públicas insostenibles ${ }^{4}$.

\section{UN TRATADO INTERGUBERNAMENTAL EN EL SENO DE LA UNIÓN EUROPEA}

Al margen de estas normas de contenido económico lo más llamativo es la vía escogida por los Estados miembros de la Unión Europea para formalizar un Tratado intergubernamental de Derecho Internacional independiente del Derecho de la Unión. Es decir, encontrándonos en el seno de la Unión Europea lo lógico hubiese sido modificar los Tratados constitutivos a través del procedimiento de revisión ordinario previsto en los artículos 48.1 a 48.5 TUE o a través del procedimiento simplificado previsto en el artículo 48.6 TUE; sin embargo, al margen de los plazos que hubiese supuesto acudir a ambos procedimientos, el principal escollo es que en los dos supuestos se exige la aprobación por todos los Estados miembros de conformidad con sus

${ }^{4}$ BCE-Boletín Mensual, Mayo 2012, pág. 99. 
respectivas normas constitucionales. Por ello, ante la falta de unanimidad de los Estados miembros, anunciada ya en la Declaración del 9 de diciembre de 2011, se ha optado por esta vía anómala en el seno de la Unión como única posibilidad para poder sacar adelante un acuerdo en materia económica y presupuestaria.

En realidad el instrumento jurídico utilizado para cerrar el Tratado de Estabilidad, Coordinación y Gobernanza en la Unión Económica y Monetaria no es más que un artificio técnico-jurídico, por tres razones básicas:

1) el nuevo Tratado únicamente es de aplicación en los Estados miembros de la zona euro de la Unión Europea que lo firmen y que posteriormente lo ratifiquen (art. 14.2 TECG);

2) a lo largo del Tratado se hace una continua referencia, como ahora veremos, a las políticas de la Unión Europea así como a las Instituciones de la misma a las cuales se atribuyen competencias concretas relacionadas con el Tratado;

3) el mismo Tratado expresa su carácter transitorio y excepcional, puesto que prevé que el contenido del mismo se incorpore en el plazo máximo de cinco años a partir de su entrada en vigor al marco jurídico de la Unión Europea (art. 16 TECG); que será interpretado de conformidad con los Tratados en los que se fundamenta la Unión Europea y con el Derecho de la Unión Europea, incluido el Derecho procesal cuando sea necesaria la adopción de Derecho derivado; y que únicamente será de aplicación en la medida en que sea compatible con los Tratados y con el Derecho de la Unión Europea (art. 2 TECG).

Una vez alcanzado el acuerdo para la firma del Tratado, el escollo de la unanimidad para la entrada en vigor del mismo queda superado al establecerse que únicamente será necesario su ratificación por 12 Estados miembros de la zona euro. Sin embargo, al tratarse de un Tratado intergubernamental nos podemos encontrar con las siguientes situaciones:

a) Que el Tratado sea ratificado por los 17 Estados miembros de la zona euro.

b) Que el Tratado no se ratifique por alguno de los 17 Estados miembros de la zona euro. En este caso se pueden plantear situaciones complejas desde el punto de vista político, económico y jurídico en el seno de la Unión Europea puesto que nos encontraríamos con Estados de la zona euro en los que regiría el nuevo Tratado y Estados de la zona euro en los que no regiría el nuevo Tratado.

A ello hay que sumar que el mismo Tratado prevé la posibilidad de que sea ratificado por Estados miembros de la Unión Europea que aunque actualmente no sean de la zona euro pasen a serlo en un futuro -en este sentido, el Tratado ha sido firmado por Bulgaria, Dinamarca, Hungría, Letonia, Lituania, Polonia y Rumanía y Suecia cuya moneda no es aún el 
euro- ${ }^{5}$; así como se prevé la posibilidad de adhesión de Estados miembros de la Unión Europea que no sean Partes Contratantes -República Checa y Reino Unido- (art. 15 TECG).

Es decir, de partida nos vamos a encontrar con tres situaciones distintas: Estados de la zona euro con nuevo Tratado, Estados fuera de la zona euro pero en camino hacia ella y Estados fuera de la zona euro sin intención, en principio, de entrar en ella. Pero además se puede dar una cuarta situación en el caso de que alguno de los 17 Estados de la zona euro no ratifique el Tratado. Todo ello va obligar a desarrollar en el seno de la Unión un complejo engranaje de negociaciones políticas, económicas y jurídicas que sobrepasarán las ya de por sí habituales dificultades que se platean normalmente en el ámbito de la Unión.

Desde un punto de vista político los primeros Estados «llamados» a ratificar el Tratado serán aquellos acuciados por los mercados y la crisis económica a los que el nuevo Tratado dará credibilidad fiscal en el ámbito internacional. En este sentido, Portugal y Grecia ya han ratificado el Tratado y es previsible que en la misma línea actúen Irlanda ${ }^{6}$ y el resto el resto de Estados mediterráneos de la zona euro (Chipre, Malta, Italia, Eslovenia y España ${ }^{7}$ ). Mientras que el resto de Estados de la zona euro cuya política económica y fiscal no ha estado sometida a presión por parte de los mercados podrían plantearse no ratificar el Tratado, especialmente en el momento en que el mismo sea ratificado por 12 Estados de la zona euro y teniendo en cuenta las obligaciones sustanciales que el Tratado conlleva.

Por otra parte, no hay que olvidar que los verdaderos actores protagonistas para que el Tratado entre en vigor son Francia y Alemania. En este sentido, a pesar de que el actual Presidente de Francia durante la campaña electoral y tras su nombramiento como Presidente había anunciado que era

${ }^{5}$ El Tratado no entrará plenamente en vigor para ellos en tanto no tengan el euro como moneda propia ya que el art. 14.4 TECG establece que «(...) el título V se aplicará a todas las Partes Contratantes de que se trate a partir de la fecha de entrada en vigor del presente Tratado». El título V es el relativo a la Gobernanza de la zona del euro y en su art. 12.3 establece que «Los Jefes de Estado o de Gobierno de las Partes Contratantes distintas de aquellas cuya moneda es el euro que hayan ratificado el presente Tratado participarán en los debates de las reuniones de la Cumbre del Euro sobre la competitividad de las Partes Contratantes, la modificación de la arquitectura general de la zona del euro y las normas fundamentales que se le aplicarán en el futuro, así como, cuando proceda y como mínimo una vez al año, en debates sobre cuestiones especificas de aplicación del presente Tratado de Estabilidad, Coordinación y Gobernanza en la Unión Económica y Monetaria».

${ }^{6}$ El 31 mayo de 2012 Irlanda celebró un referéndum en que el 60,3\% de los votantes se manifestaron a favor de ratificar el Tratado.

${ }^{7}$ Las Cortes Generales han aprobado la Ley Orgánica 3/2012, de 25 de julio, por la que se autoriza la ratificación por España del Tratado de Estabilidad, Coordinación y Gobernanza en la Unión Económica y Monetaria. Por otra parte, España con el fin de dar mayor confianza internacional, económicamente hablando, reformó el artículo 135 de su Constitución en septiembre de 2011. 
necesario renegociar el Tratado ${ }^{8}$, el 10 de octubre de 2012 la Asamblea Nacional francesa ratificó el mismo, por lo que desaparece uno de los principales escollos para la entrada en vigor del Tratado, pues la no ratificación de Francia hubiese supuesto prácticamente la muerte del mismo.

\section{EL TECG EN RELACIÓN AL DERECHO E INSTITUCIONES DE LA UNIÓN EUROPEA}

Como hemos señalado al inicio, a pesar de ser un Tratado de carácter intergubernamental y por tanto estar inserto en el ámbito del Derecho Internacional contiene continuas referencias a la normativa y política de la Unión Europea. Así, por ejemplo, se afirma que «serán de aplicación las definiciones establecidas en el artículo 2 del Protocolo $\left(n^{\circ} 2\right)$ sobre el procedimiento aplicable en caso de déficit excesivo, anejo a los Tratados de la Unión Europea» (art. 3.3 TECG); «la existencia de un déficit excesivo debida al incumplimiento del criterio de la deuda se decidirá de conformidad con el procedimiento establecido en el artículo 126 del Tratado de Funcionamiento de la Unión Europea» (art. 4 TECG); «sobre la base de la coordinación de las políticas económicas definida en el Tratado de Funcionamiento de la Unión Europea, las Partes Contratantes se comprometen a cooperar en aras de la política económica que fomente...» (art. 9 TECG).

Además se prevé la puesta en marcha de actuaciones propias de la Unión Europea para conseguir los objetivos fijados. En este sentido, el artículo 10 hace referencia expresa a la cooperación reforzada que conlleva que los Estados miembros que lo deseen podrán instaurar entre sí una cooperación reforzada con el fin de impulsar los objetivos de la Unión y proteger sus intereses (art. 20 TUE). No obstante, la cooperación reforzada no puede utilizarse en el ámbito de las competencias exclusivas (art. 20. 1 TUE) ni tampoco puede perjudicar al mercado interior (art. 326 TFUE); por ello, el Tratado establece que la cooperación reforzada se ponga en marcha sobre cuestiones que sean esenciales para el correcto funcionamiento de la zona euro, de modo que en este caso únicamente podrá emplearse con carácter residual y respecto a aspectos muy concretos, dado que la política monetaria es competencia exclusiva de la Unión (art. 3.1 c TFUE) y puede afectar a reglas del mercado interior.

Por otro lado, como nos encontramos ante un artificio técnico-jurídico que no pretende sino ser aplicado de forma exclusiva a determinados Estados miembros de la Unión Europea, el mismo Tratado contiene una cláusula expresa de subordinación al Derecho de la Unión. Así, el art. 2 TECG establece que el Tratado será interpretado de conformidad con los Tratados

${ }^{8}$ En mayo de 2012 se celebraron en Francia las Elecciones Presidenciales resultando elegido François Hollande frente a Nicolas Sarkozy. 
en los que se fundamenta la Unión Europea y con el Derecho de la Unión Europea, incluido el Derecho procesal cuando sea necesaria la adopción de Derecho derivado; y que únicamente será de aplicación en la medida en que sea compatible con los Tratados y con el Derecho de la Unión Europea.

Junto a ello, el Tratado atribuye competencias a instituciones de la Unión Europea, algo también difícil de insertar en una estructura de raíz intergubernamental dado el interés supranacional de estas instituciones; por ello resulta complicado pensar cómo actuarán a la vez en un sistema comunitario de 27 y en otro intergubernamental de 25 (o menos). Las instituciones europeas referidas expresamente son: el Consejo de la Unión Europea, la Comisión Europea y el Tribunal de Justicia de la Unión Europea ${ }^{9}$. Llama, sin embargo, la atención que el nuevo Tratado rompa con la tendencia instaurada por el Tratado de Lisboa de dar protagonismo al Parlamento Europeo dado que no atribuye competencia alguna al mismo, salvo dos funciones de carácter menor que veremos en el siguiente punto.

En todo caso, el Tratado atribuye a la Comisión Europea proponer el calendario para lograr la convergencia de equilibrio presupuestario a medio plazo, proponer los principios comunes del mecanismo corrector que deberá aplicarse en caso de que se observen desviaciones significativas en el objetivo de déficit establecido, así como formular propuestas o recomendaciones en el supuesto de incumplimiento del déficit (arts. 3 y 7 TECG). Además las Partes Contratantes deberán presentar un programa de colaboración presupuestaria y económica que incluya las reformas estructurales que deben adoptar; dicho programa deberá ser aprobado y supervisado en su ejecución por el Consejo de la Unión Europea y la Comisión Europea (art. 5 TECG).

En caso de incumplimiento de lo previsto en el art. $3.2^{10}$ la Comisión Europea podrá realizar un informe y brindar a la Parte Contratante afectada la oportunidad de presentar observaciones; al margen del informe final de la

${ }^{9}$ El artículo 11 del TECG relativo a la coordinación de las políticas económicas contiene una cláusula general en este sentido al afirmar que «participarán en tal coordinación las instituciones de la Unión Europea según lo requiera el Derecho de la Unión Europea».

${ }^{10} \mathrm{El}$ art. 3.2 TECG establece que: «Las normas establecidas en el apartado 1 se incorporarán al Derecho nacional de las Partes Contratantes a más tardar un año después de la fecha de entrada en vigor del presente Tratado mediante disposiciones que tengan fuerza vinculante y sean de carácter permanente, preferentemente de rango constitucional, o cuyo respeto y cumplimiento estén de otro modo plenamente garantizados a lo largo de los procedimientos presupuestarios nacionales. Las Partes Contratantes establecerán a escala nacional el mecanismo corrector mencionado en el apartado 1, letra e), sobre la base de los principios comunes que proponga la Comisión Europea, relativos en particular a la naturaleza, el alcance y el calendario de la medida correctora que se adopte, igualmente en caso de circunstancias excepcionales, y a la función e independencia de las instituciones responsables a escala nacional de supervisar la observancia de las normas establecidas en el apartado 1. Dicho mecanismo corrector respetará plenamente las prerrogativas de los Parlamentos nacionales». 
Comisión, una o más Partes Contratantes podrán someter el asunto al Tribunal de Justicia de la Unión Europea, cuya Sentencia será vinculante. En caso de no ejecución de la Sentencia se podrá someter el asunto al Tribunal de Justicia quien podrá imponer sanciones de carácter pecuniario (art. 8 TECG).

\section{LA GOBERNANZA DE LA ZONA DEL EURO}

Un elemento importante del Tratado es la mejora de la gobernanza de la zona del euro ${ }^{11}$. El nuevo Tratado formaliza la celebración de las Cumbres del Euro al menos dos veces al año. Además se prevé que los Jefes de Estado o de Gobierno de las Partes Contratantes cuya moneda es el euro celebren de manera informal reuniones de la Cumbre del Euro junto con el Presidente de la Comisión Europea, a las que se invitará al Presidente del Banco Central Europeo.

El Presidente de la Cumbre del Euro será designado por mayoría simple por los Jefes de Estado o de Gobierno de las Partes Contratantes cuya moneda es el euro, al mismo tiempo que el Consejo Europeo elija a su Presidente y para un mandato de igual duración. El Presidente de la Cumbre del Euro garantizará la preparación y continuidad de las reuniones de la Cumbre, en estrecha cooperación con el Presidente de la Comisión Europea, y presentará un informe al Parlamento Europeo después de cada reunión de la Cumbre del Euro.

El reconocimiento formal de las reuniones de la Cumbre del Euro contribuye a promover el compromiso y la responsabilidad para el correcto funcionamiento de la zona del euro al más alto nivel político. La asistencia de los Jefes de Estado o de Gobierno de los respectivos Estados permitirá que las orientaciones políticas acordadas en dichas reuniones puedan incluir áreas que vayan más allá del ámbito de los Ministerios de Economía y Finanzas, lo que facilitará la coordinación de todas las áreas e instrumentos políticos relevantes necesarios para el adecuado funcionamiento de la zona euro ${ }^{12}$.

Por otra parte, los Jefes de Estado o de Gobierno de las Partes Contratantes distintas de aquellas cuya moneda es el euro que hayan ratificado el Tratado participarán en los debates de las reuniones de la Cumbre del Euro sobre la competitividad de las Partes Contratantes, la modificación de la ar-

${ }^{11}$ En las reuniones de la Cumbre del Euro celebradas en 2011 ya se tomaron una serie de decisiones para reforzar la gobernanza de la zona del euro. Estos elementos, que no se han incorporado al TECG, incluyen: i) la ampliación de las capacidades de decisión de la zona del euro, principalmente a través de un fortalecimiento permanente de las estructuras de preparación y de la elección de un presidente del grupo de trabajo del Eurogrupo con sede en Bruselas y con dedicación a tiempo completo; y ii) la celebración de reuniones mensuales de los presidentes de la Cumbre del Euro, la Comisión y el Eurogrupo (Véase el anexo I de la declaración de la Cumbre del Euro de 26 de octubre de 2011 («Diez medidas para mejorar la gobernanza de la zona del euro»).

${ }^{12}$ BCE-Boletín Mensual, Mayo 2012, pág. 96. 
quitectura general de la zona del euro y las normas fundamentales que se le aplicarán en el futuro, así como, cuando proceda y como mínimo una vez al año, en debates sobre cuestiones específicas de aplicación del Tratado.

Finalmente, se establece que el Parlamento Europeo y los Parlamentos nacionales de las Partes Contratantes decidirán conjuntamente la organización y la promoción de una conferencia de representantes de las comisiones pertinentes del Parlamento Europeo y de las de los Parlamentos nacionales, a fin de debatir políticas presupuestarias y otras cuestiones del ámbito del nuevo Tratado.

\section{CONCLUSIONES}

Tras la celebración del Consejo Europeo de diciembre de 2011 se puso de manifiesto la falta de unanimidad de los Estados miembros de la Unión Europea para alcanzar un pacto de estabilidad fiscal. Debido a la grave crisis económica existente y el escollo que suponen las exigencias procedimentales establecidas en los Tratados de la Unión Europea, veinticinco Estados miembros de la misma acordaron firmar un Tratado intergubernamental de Estabilidad, Coordinación y Gobernanza en la Unión Económica y Monetaria; dicho Tratado constituye un paso adelante hacia un marco de regulación presupuestaria basado en reglas estrictas como la introducción obligatoria del equilibrio presupuestario y del mecanismo corrector automático a escala nacional.

Sin embargo, la entrada en vigor de dicho Tratado intergubernamental plantea importantes incertidumbres políticas, económicas y jurídicas en el seno de la Unión al tratarse de un artificio jurídico ideado para salvaguardar la estabilidad económica de la Unión Europea.

A ello hay que sumar la inestable situación política y económica en que se encuentran varios Estados de la zona euro que incluso aunque alguno de ellos ya ha ratificado el Tratado -Grecia-cada día se especula más con su salida del euro; así como la situación política de Francia que acaba de elegir nuevo Presidente y que mantiene la posición de que es necesario renegociar este Tratado. 